\title{
LA CALIDAD EDUCATIVA EN LA FORMACIÓN DE POSGRADO EN BIOÉTICA. EL PROGRAMA DE MAESTRÍA EN BIOÉTICA DE LA UNIVERSIDAD NACIONAL DE CUYO
}

\author{
Marta Fracapani Cuenca de Cuitiño , Fernando Lolas Stepke ${ }^{* *}$ y Marisa Carina Fazio ${ }^{* * *}$
}

\begin{abstract}
Resumen: El presente documento propone la evaluación de programas como una herramienta necesaria para garantizar la calidad de la formación de la enseñanza, y expone la experiencia evaluativa realizada con el curso de Bioética Fundamental, uno de los dos que constituye el programa de Maestría en Bioética ofrecido por la Universidad Nacional de Cuyo, en Mendoza, Argentina. Sugiere, además, un conjunto de desafíos impuestos por la compatibilidad, comparabilidad y competitividad de las diferentes ofertas educativas que surjan en América Latina y el Caribe.
\end{abstract}

Palabras clave: formación de posgrado en bioética, evaluación de la calidad de programas en bioética, bioética, implicancias educativas de la globalización

\section{EDUCATIONAL QUALITY WHILE DICTATING A POSTGRADUATE DEGREE ON BIOETHICS. CUYO'S NATIONAL UNIVERSITY'S MASTER'S DEGREE ON BIOETHICS.}

\begin{abstract}
This paper intends. to evaluate degrees as a necessary tool in order to assure good quality while teaching a postgraduate course. It sets forth the evaluative experience offered together with the class on Fundamental Bioethics, one of the two courses that conform the Master's degree on Bioethics offered by Cuyo's National University, in Mendoza, Argentina.

It also suggests a number of challenges necessary for compatibility, comparability and competitivity of the different educational opportunities offered throughout Latin America and the Caribbean
\end{abstract}

Key words: postgraduate education on bioethics, evaluation of bioethics degrees' educational quality, bioethics, meaningful educational results of globalization

\section{A QUALIDADE EDUCATIVA NA FORMAÇÃO DE PÓS-GRADUAÇÃO EM BIOÉTICA. O PROGRAMA DE MESTRADO EM BIOÉTICA DA UNIVERSIDADE NACIONAL DE CUYO}

Resumo: O presente documento propõe a avaliação de programas como uma ferramenta necessária para garantir a qualidade da formação do ensino e expõe a experiência de avaliação realizada no curso de bioética fundamental, um dos que constitui o programa de mestrado em bioética oferecido pela Universidade Nacional de Cuyo, em Mendonza, Argentina. Sugere, além disso, um conjunto desafios impostos pela compatilibidade, comparabilidade e competitividade das diferentes ofertas educativas que surgem na América Latina e Caribe.

Palavras chave: formação em pós-graduação em bioética, avaliação da qualidade de programas de bioética, bioética, implicações educativas da globalização

\footnotetext{
Directora de la Maestría en Bioética. Facultad de Ciencias Médicas, Universidad Nacional de Cuyo. Mendoza. Argentina

** Profesor Titular de la Universidad de Chile. Director de la Unidad de Bioética, OPS/OMS

*** Profesora. Magíster. Becaria Doctoral del CONICET. Argentina

Correspondencia: mfracapani@millic.com.ar
} 


\section{Antecedentes del programa}

El programa de Maestría en Bioética que se desarrolla en la Universidad Nacional de Cuyo (Mendoza, Argentina) nació como una estrategia necesaria para incrementar la masa crítica que se requería para la incorporación de la bioética como contenido en el diseño curricular de grado y posgrado de distintas disciplinas. Surge de una alianza estratégica entre la Facultad de Ciencias Médicas de la Universidad Nacional de Cuyo y el Programa Regional de Bioética para América Latina y el Caribe OPS/ OMS, hoy Unidad de Bioética, con sede en Santiago de Chile. Está orientado a proporcionar una formación sistemática, inter y transdisciplinaria, a distintos profesionales interesados en el estudio del hombre y su medio ambiente, con variedad de metodologías y en un marco pluralista, democrático y en diálogo crítico.

Los antecedentes de este proyecto responden a iniciativas de nivel internacional, nacional y regional en las actuales políticas de educación superior universitaria.

En Argentina la situación de la educación superior no difiere mayormente de la encontrada en el resto de las universidades latinoamericanas y caribeñas. Sus fortalezas y debilidades son semejantes, por ejemplo, en las relaciones con los Estados, con la sociedad en general, con las empresas, con las organizaciones sindicales y los estudiantes. La educación superior universitaria ha tenido un notable incremento en el nivel del pregrado pero mucho menor en la formación de posgrado. En general, las restricciones financieras de las universidades públicas son comunes en muchos de los países, al igual que las demandas de nuevos perfiles profesionales que respondan con conocimientos, habilidades y actitudes no requeridas hasta ahora. El contexto mundial se caracteriza por la internacionalización y el establecimiento de alianzas y redes globales; la in- corporación de la educación virtual no presencial; la convergencia digital de industrias culturales y servicios educacionales; la globalización económica; el fuerte desarrollo del conocimiento científico y el nacimiento de redes.

Para la formación de bioeticistas se analizó el actual sistema educativo en escuelas de Medicina y se verificó que:

- Los egresados durante la formación de pregrado han adquirido un nivel técnico y científico acorde con los avances acaecidos mundialmente; sin embargo, poseen escasas herramientas para afrontar en forma significativa los problemas éticos, sociales y legales: problemas que inciden en la salud del hombre, en sus derechos individuales y colectivos, en la sociedad y el medio ambiente.

- Existe un desequilibrio curricular entre la enseñanza de los valores y de las ciencias. Es frecuente que, de tales sistemas de formación, egresen tecnólogos sin valores humanísticos, incapaces de pensar éticamente el obrar científico-técnico y de analizar sus consecuencias en la persona humana.

- A esto se suman los nuevos modelos de relación entre el equipo de salud y el paciente, así como los requerimientos éticos que plantea la investigación científica o la gestión de los servicios de salud.

Es imprescindible que los profesionales reflexionen sobre su accionar, teniendo como referente el valor de la vida humana. De igual modo se impone analizar la asignación de recursos. El principal aporte de la bioética consiste en considerar no sólo los hechos clínicos en el proceso de toma de decisiones, sino también los valores de los pacientes, de los profesionales y de la sociedad. 


\section{Objetivos y metas del programa}

Considerando como fundamento las demandas expuestas hasta el momento, los objetivos de este posgrado son:

- Generar un espacio adecuado de deliberación social en el respeto por la democracia, para optar éticamente respecto de los desafíos que plantea el desarrollo acelerado de la tecnociencia.

- Impulsar la elaboración y difusión de normativas jurídicas que promuevan los derechos y deberes de las personas.

- Mejorar la atención y el cuidado de la salud de la población potenciando los aspectos éticos.

- Formar expertos y consultores en bioética, capaces de gestionar, de modo eficaz y eficiente, los comités hospitalarios de ética y los de ética de investigación, y propiciar la creación de comités de atención primaria de la salud.

- Mejorar la docencia de pre y posgrado en programas de formación en bioética.

- Desarrollar la investigación en el área.

- Facilitar la formación de recursos humanos que estructuren un sistema de salud efectivo y equitativo, que desarrolle acciones técnicamente apropiadas, políticamente factibles y éticamente sustentables.

Partiendo de lo expuesto, y teniendo en cuenta la complejidad y relevancia que ha adquirido la bioética en los últimos tiempos, este proyecto busca:

- Ofrecer una formación sistemática multi, trans e interdisciplinaria, en un ámbito como el de la ciencia y de la salud que actualmente requiere de grandes respuestas.
- Mejorar la atención y el cuidado de la salud mediante la intervención de la actitud ética del médico y de otros profesionales frente a la vida humana.

Para ello, el perfil del egresado es el de un profesional que sea capaz de:

- Detectar los aspectos éticos inherentes a cada una de las profesiones.

- Resolver en forma argumentada los conflictos éticos que aparezcan en su práctica profesional, tanto en el plano local como en el provincial, nacional e internacional.

- Conocer los comités de ética, sus tipos y funcionamiento, las dificultades y los modos de ser consultados.

- Conocer y comprender la historia, fundamentación y metodología de la ética aplicada.

- Integrar y/o gestionar los diversos tipos de comités de ética.

- Trabajar en equipos inter y transdisciplinarios en forma civil, pluralista, racional, aplicada, reguladora y procedimental.

- Acceder a la información sobre bioética, a partir del uso significativo de las diferentes fuentes.

- Utilizar documentación y otros recursos sobre bioética (leyes, decretos, reglamentos, obras artísticas y medios audiovisuales).

- Investigar en el campo de la bioética con el fin de realizar aportes significativos a la comunidad.

- Programar y desarrollar tareas docentes para mejorar la formación de los actuales profesionales. 
- Actuar éticamente en forma individual o en un equipo en el campo de la salud.

- Asumir la responsabilidad de la ética.

\section{Características del programa}

La Universidad Nacional de Cuyo ha mantenido las características generales del diseño de otras universidades, aunque introduciendo significativas reformas. Este diseño permite, además, la incorporación de alumnos que ya han realizado algunos módulos en universidades extranjeras, generando un nuevo modelo de articulación e integración latinoamericana. Es también la primera experiencia desarrollada en su totalidad por profesores latinoamericanos y la primera maestría de carácter internacional para nuestra unidad académica.

Tiene una carga horaria total de 600 horas reales dictadas y 160 horas de tutoría y tareas de investigación, todo ello estructurado en dos cursos: uno de Bioética Fundamental y otro de Bioética Clínica, con 280 horas presenciales y 20 horas de cursos electivos cada uno.

\section{Propósitos de este documento}

El presente documento apunta a describir y emitir juicios de valor sobre el curso de Bioética Fundamental, desarrollado entre el 5 de abril y el 23 de mayo de 2005 en la Facultad de Ciencias Médicas de la Universidad Nacional de Cuyo.

La estructura lógica utilizada para que este estudio cumpla con los objetivos explicitados con anterioridad es la siguiente:

- Justificación y objetivos de la evaluación.

- Metodología de evaluación utilizada.

- Aspectos del programa de formación valorados. Valoración.
Concebimos la evaluación como una estrategia y una herramienta valiosa de la gestión educativa, pues permite:

- Identificar, mediante la descripción exhaustiva de cada uno de los elementos que conforman el sistema de formación, las falencias por superar y las fortalezas por potenciar.

- Comprender el comportamiento de dichas variables y emitir juicios de valor al respecto.

- Proponer estrategias de mejora y/o innovación.

Los procesos de globalización y regionalización exigen calidad educativa a los actuales programas de formación, convirtiéndose así en la meta hacia la cual apunta la actual política educativa en formación de posgrado en los distintos países.

De acuerdo a Blas y otros, la calidad es: "Un constructo, una variable multidimensional y compleja que requiere de una pluralidad de componentes: efectividad, eficiencia, eficacia, relevancia y toda otra variable que, para determinada circunstancia, forme parte del concepto de calidad. Por ello, es un constructo relativo, ya que solo es un término de referencia comparativo respecto a un modelo elaborado para un determinado contexto( $(1)$ ".

Para una mejor comprensión del concepto de calidad, es necesario aclarar que la efectividad es la congruencia entre los objetivos declarados y los resultados obtenidos, y la eficiencia se refiere al rendimiento interno de la institución, al mejor uso posible de los recursos y del tiempo, de acuerdo con las funciones y misiones preestablecidas para y por la institución. La eficacia, en cambio, hace referencia al rendimiento externo; se relaciona directamente con el impacto social que sus resultados producen. La relevancia, finalmente, es el gra- 
do de adecuación de la oferta educativa y productiva a los requerimientos prioritarios de la sociedad.

Estos conceptos o variables se agrupan para constituir el concepto medular de calidad: la congruencia de todos los requerimientos de una institución con los valores educativos, con los objetivos científicos, con las demandas de la sociedad y con la misión específica que asume cada institución.

La Maestría en Bioética se ha propuesto prestar mucha atención al estudio de los determinantes de la salud, al logro de la equidad en la asistencia y los servicios, y a la mejora de la calidad de vida. Para cumplir con dichas características apostamos a la evaluación como estrategia que orienta la toma de decisiones para el mejoramiento, mantenimiento de la calidad e/o innovación de la oferta educativa. Todo este proceso, expresado en juicios valorativos, contribuirá a garantizar la calidad del plan de formación mediante el diseño de estándares de desempeño y pedagógico-didácticos.

\section{Metodología del proceso de evaluación}

Teniendo en cuenta el concepto de calidad y la actual política educativa -nacional e internacional-, la evaluación de programas de formación es un: "Proceso con carácter constructivo, participativo y consensuado; es una práctica permanente y sistemática que permite detectar los nudos problemáticos y los aspectos positivos. Ello implica tanto los aspectos cualitativos como cuantitativos, con un alcance que abarca los insumos, los procesos, los productos y el impacto que tienen en la sociedad; una tarea fundamental para el gobierno y la gestión administrativa y académica; $y$, en definitiva, una plataforma para el planeamiento institucio$\operatorname{nal}(2)$ ".

Partiendo de esta definición los tres propó- sitos evaluativos son:

- Proporcionar datos para la responsabilidad.

- Promover la comprensión de los fenómenos implicados.

- Servir de guía para la toma de decisiones.

Teniendo en cuenta los actuales enfoques evaluativos y los lineamientos de evaluación establecidos por la Comisión Nacional de Evaluación y Acreditación Universitaria (CONEAU)(3), a partir de la Ley de Educación Superior, el proceso de evaluación debe contar con instancias internas y externas. $\mathrm{La}$ evaluación interna tiene por objeto analizar los logros y dificultades en el cumplimiento de las funciones, así como las estrategias de mejora e innovación producto de dicha valoración. La evaluación externa complementa y enriquece a la primera(4).

Cabe resaltar que el programa aún no ha sido presentado para acreditación ante la CONEAU debido a que, desde 2002, no se ha realizado ninguna convocatoria nacional. No obstante, cuenta con un plan de evaluación sistémico y sistemático, que abarca todas las dimensiones habituales de un programa de formación y cada una de las etapas de diseño y gestión de los cursos que forman parte de dicha maestría. Por tal motivo, se han llevado a cabo instancias paralelas y complementarias de autoevaluación y evaluación externa.

Teniendo en cuenta el modelo de evaluación que subyace en nuestro plan -que se refleja en el enfoque C.I.P.P. de D. Stufflebeam- podemos decir que, hasta el momento, hemos realizado evaluaciones diagnósticas para diseñar y reformular el programa de formación, y evaluaciones formativas para mejorar continuamente la calidad de la oferta educativa; pretendemos, de este modo, adaptarla a las actuales demandas educativas y científicas de América 
Latina y el Caribe. La evaluación final de producto, que consistirá en medir y valorar el impacto social-científico y educativo del programa, se llevará a cabo a partir del desarrollo e implementación de las tesis de los profesionales y del seguimiento de los egresados en sus puestos de trabajo. Allí, a partir de la transferencia de lo aprendido, podrán convertir sus aprendizajes en transformaciones sociales, indicadores reales del impacto de nuestro trabajo de formación.

\section{Aspectos considerados en la evaluación}

a) Desde el punto de vista de la gestión y organización, identificar, valorar y tomar decisiones sobre aspectos tales como:

- Convocatoria (becas) y cantidad de alumnos.

- Sistema de difusión de la oferta educativa.

- Sistema de curso intensivo.

- Ingreso de población estudiantil latinoamericana.

b) Desde el punto de vista formativo:

- Presentar el comportamiento de las diferentes variables curriculares: programas de estudio, mediación pedagógica, recursos didácticos, perfil de los formadores, características de los educandos, entre otras.

- Identificar y valorar dichas variables con el objeto de potenciar las fortalezas y superar las debilidades.

- Brindar la información necesaria para la toma de decisiones en pos de la mejora de la calidad y/o innovación del actual plan de estudio.
- Resaltar la importancia de la formación de un profesional capaz de liderar el desarrollo sustentable de Latinoamérica.

c) Desde el punto de vista de la extensión y transferencia:

- Demostrar la viabilidad de la articulación universidad-comunidad, a través de una institución gubernamental y de la participación en proyectos de investigación interinstitucionales.

- Identificar el aporte significativo a la formación del actual perfil del bioeticista y de recursos humanos relacionados con la temática.

- Detectar el impacto en la formación de pregrado, a partir de la formación de recursos humanos en bioética a cargo de los egresados.

El modelo valora los programas de formación en forma holística y flexible. Se ha buscado describir e interpretar las situaciones que contribuyen a la formación, con el objeto de brindar información significativa para la toma de decisiones, tanto entre los receptores de la evaluación y sujetos de formación como entre los responsables de su gestión y administración.

La metodología utilizada fue cuantitativa y cualitativa por la naturaleza del objeto de evaluación, que refleja la complejidad de todo acto educativo y del propósito de la evaluación.

\section{Resultados y discusión}

Aspectos del programa de formación valorados. Valoración. 
Acta Bioethica 2005; 11 (2)

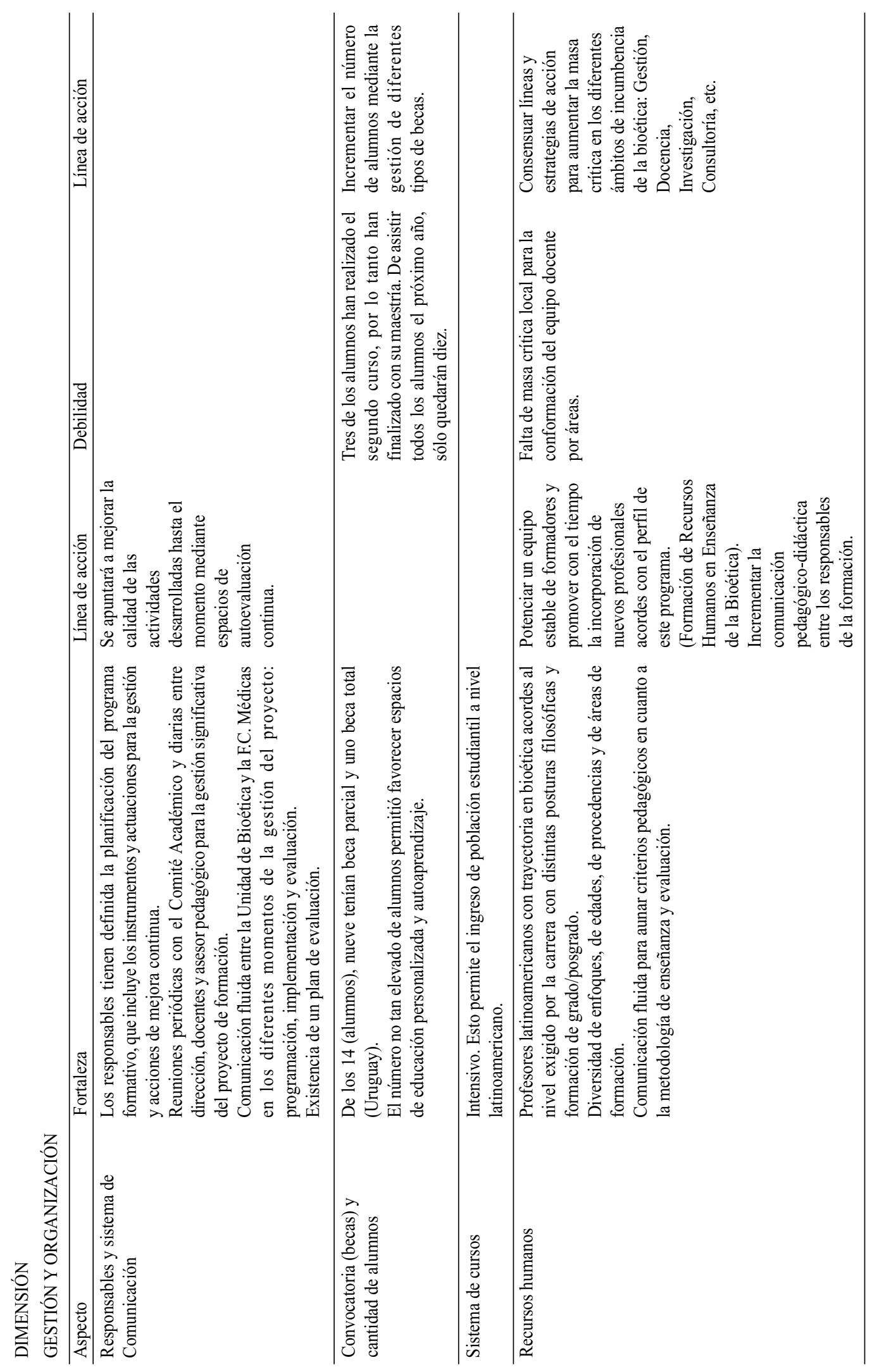


La calidad educativa en la formación de posgrado en bioética - M. Fracapani, F. Lolas y M. Fazio

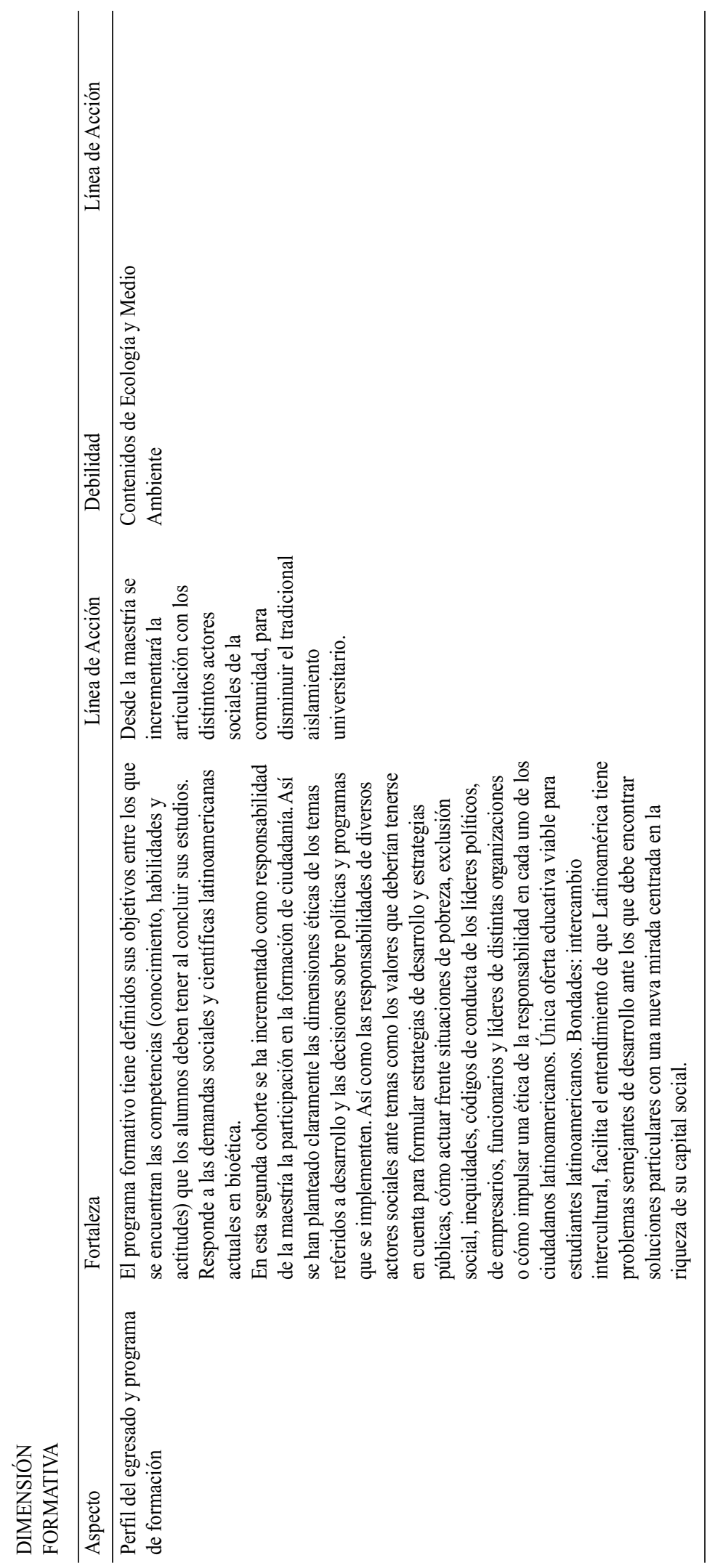


Acta Bioethica 2005; 11 (2)
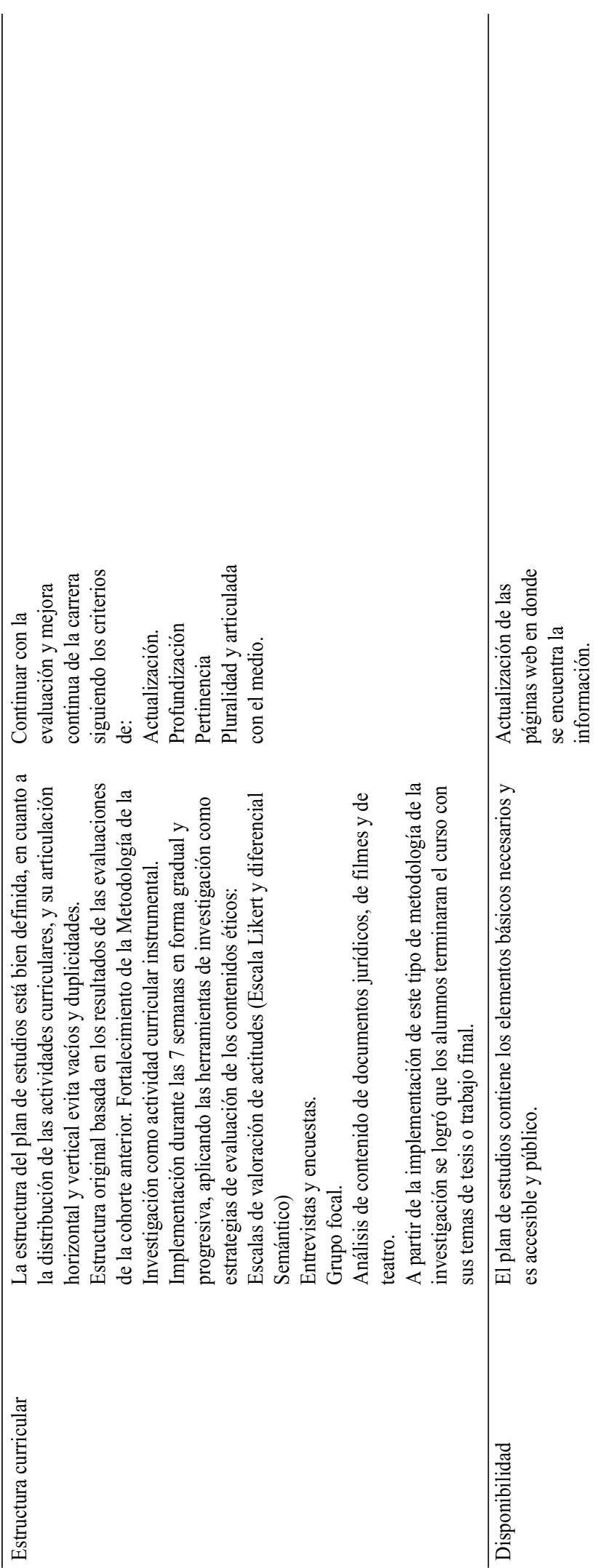


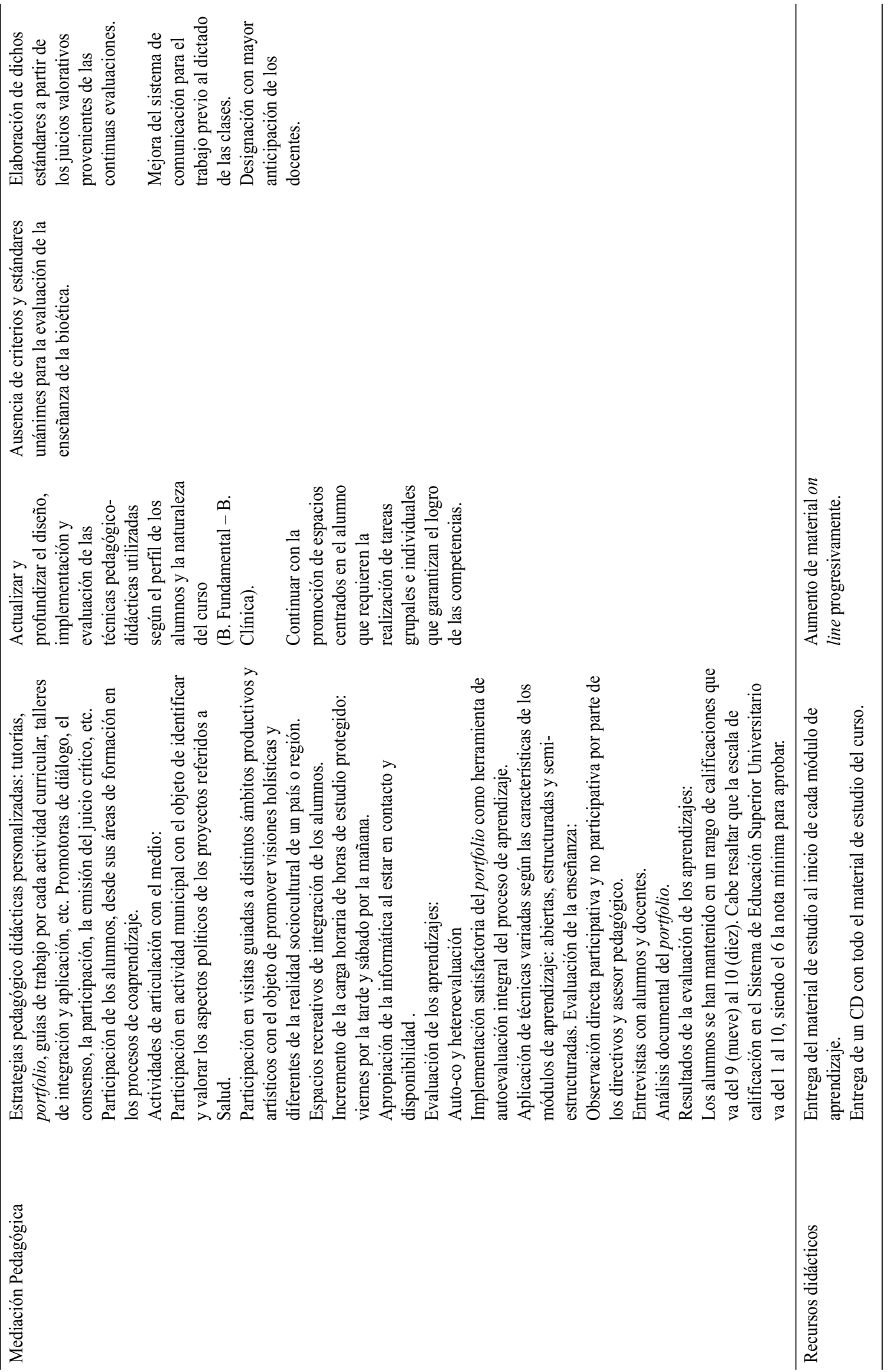


Acta Bioethica 2005; 11 (2)

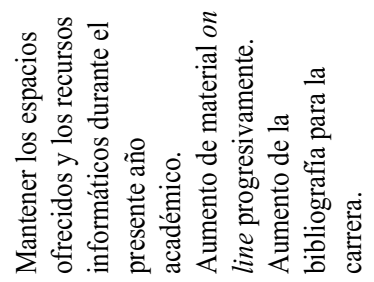

굴

응 음

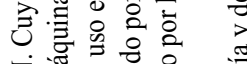

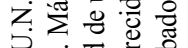

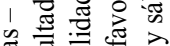

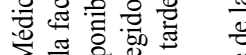

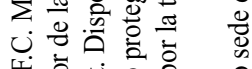

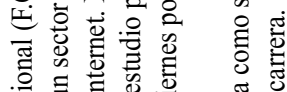

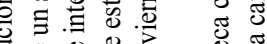

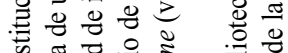

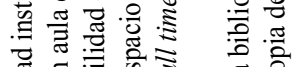



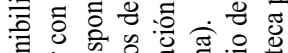

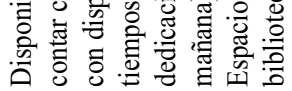

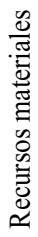


DIMENSIÓN DE EXTENSIÓN Y TRANSFERENCIA (Actividades desarrolladas dentro del ámbito del programa).

- Integración de parte del equipo técnico que logró la acreditación del Municipio de Guaymallén, de la Provincia de Mendoza, como Municipio Saludable.

- Participación en la integración del Comité de Ética de la Investigación del Hospital Dr. Humberto Notti (Mendoza).

- Participación en la integración del Comité de Bioética del Hospital Dr. Humberto Notti (Mendoza).

DIMENSIÓN CIENTÍFICA (Proyectos de investigación con evaluación de organismos científicos y del Ministerio de Salud de la Nación).

- Formación de redes interinstitucionales y transdisciplinarias al servicio de la educación para la salud (Secretaría de Ciencia y Técnica del Ministerio de Educación, Ciencia y Tecnología de la Nación, 2003-04).

- Inequidades en seguridad vial (Proyecto Vigía-Ministerio de Salud de la Nación).

- La evaluación como estrategia de mejora de la calidad e/o innovación de la Maestría en Bioética (tesis doctoral, CONICET).

\section{Desafíos}

- Lograr el desarrollo de titulaciones fácilmente comparables y comprensibles en una forma articulada en toda América Latina y el Caribe.

- Desarrollar perfiles profesionales en términos de competencias incluyendo destrezas, conocimientos y actitudes.

- Impulsar la innovación a través de comunicación de experiencias y la identificación de buenas prácticas.

- Crear redes para lograr innovación y calidad mediante la reflexión y el intercambio mutuo.

- Crear puentes entre las universidades y otras instituciones calificadas para elaborar metodologías que permitan analizar los elementos comunes de las titulaciones, las áreas específicas y diversas y encontrar la forma de alcanzar consensos.

- Lograr acuerdos sobre los mecanismos de evaluación.

- Encontrar métodos y criterios de evaluación comparables.

- Trabajar y estimular el desarrollo de distintas redes temáticas de investigación bioética que permitan a Latinoamérica acercarse a las metas del milenio.

\section{Referencias}

1. Blas I, et al. Evaluación Institucional de Unidades Académicas pertenecientes a la Universidad Nacional de Cuyo. Mendoza: Facultad de Filosofía y Letras, U. N. de Cuyo; 1996: 82.

2. Colás Bravo P, Rebollo Catalán MA. Evaluación de programas. Una guía práctica. Sevilla: Kronos; 1993.

3. CONEAU. Lineamientos para la Evaluación Institucional. Buenos Aires: Ministerio de Cultura y Educación; 1998. 
4. CONEAU. Procedimientos, Criterios y Solicitud de Acreditación de Carreras y Proyectos de Posgrado. Buenos Aires: Ministerio de Cultura y Educación; 2002.

\section{Bibliografía}

Álvarez A, Del Río P. Educación y desarrollo de la teoría de Vigotsky. Madrid: Alianza; 1992: 93-120.

Asociación de Bioética Fundamental y Clínica. La educación en Bioética de los Profesionales Sanitarios en España. Una propuesta organizativa. Madrid: Asociación de Bioética Fundamental y Clínica; 1999.

Apostel L, Benoist JM, Bottomore T, et al. La interdisciplinariedad en las ciencias sociales. Madrid: Tecnos UNESCO; 1982.

Coll C. Aprendizaje escolar y construcción del conocimiento. Buenos Aires: Paidós; 1991: 189-206.

Cortina A. El mundo de los valores. Ética mínima y educación. Santa Fe de Bogotá: El Búho; 2000.

Challis M. Portfolio and assessment: meeting the challenge. Med. Teach. 2001; 23(5): 437-440.

Friedman M. AMEE Guide N 24 Portfolios as a method of students assessment. Med. Teach. 2001, 23(6): 531-551.

Friedman C, De Blick R, Greer D, et al. Charting the winds of change: Evaluating innovative medical curricula. Special article. Acad. Med. 1990: 65: 88-14.

Gracia D. Fundamentación y enseñanza de la bioética. Santa Fe de Bogotá: El Búho; 1998: 175-183.

Lolas F. Bioethics. Moral in life sciences. Santiago de Chile: Editorial Universitaria; 1999.

Mainetti J. La transformación de la Medicina. La Plata: Quirón; 1992.

Palacios J, Marchsi A, Coll C. Desarrollo psicológico y educación. Psicología Evolutiva. Madrid: Alianza; 1992.

Piaget J. Adaptación vital y sociológica de la inteligencia. Buenos Aires: Siglo XXI; 1985: 117-190.

Prieto Castillo D. La enseñanza en la Universidad. Mendoza: EDIUNC; 1995.

World Federation on Medical Education. Quality Improvement in Basic Medical Education. WFME. International Guidelines. Denmark: University of Copenhagen; October 2001.

Universidad Nacional de Cuyo. Evaluación Institucional. Mendoza: EDIUNC; 1996.

Velasco F. Presupuestos éticos de una sociedad plural en la pregunta por la ética. Salamanca: Universidad Pontificia de Salamanca; 1993: 162-181.

Venturelli J. Educación médica: nuevos enfoques, metas y métodos. Washington DC: OPS/OMS; 1995.

XVI Conferencia Panamericana de Educación Médica. Autoevaluación y Acreditación en tiempo de cambio. Educación Médica 2003; 6(S1): 59-63. 